https://doi.org/10.15407/ujpe64.7.589

D. HORVÁTH

Wigner Research Centre for Physics, Budapest, Hungary;

and Institute of Nuclear Research (Atomki), Debrecen, Hungary

(H-1121 Budapest, Konkoly-Thege 29-33, Hungary; e-mail: horvath.dezso@wigner.mta.hu,

dezso.horvath@cern.ch)

\title{
TESTS OF CPT INVARIANCE AT THE ANTIPROTON DECELERATOR OF CERN
}

\begin{abstract}
The Standard Model, the theory of particle physics is based on symmetries: both the structure of the composite particles and their interactions are derived using gauge invariance principles. Some of these are violated by the weak interaction like parity and CP symmetry, and even masses are created via spontaneous symmetry breaking. CPT invariance, the most essential symmetry of the Standard Model, states the equivalency of matter and antimatter. However, because of the lack of antimatter in our Universe it is continuously tested at CERN. We overview these experiments: measuring the properties of antiprotons as compared to those of the proton at the Antiproton Decelerator and also searching for antimatter in cosmic rays.
\end{abstract}

Keywords: Standard Model, CPT invariance, antiproton mass, antihydrogen, cosmic antimatter.

\section{Introduction}

The theory behind particle physics, called for historic reasons the Standard Model, developed half a century ago, is based on gauge symmetries [1]. Some of those, however, are violated, like the maximally broken parity symmetry or the tiny little $C P$-violation. And of course, there is the spontaneous symmetry breaking mechanism necessary to create masses for the elementary particles.

The fundamental particles of the Standard Model are fermions with half-integer and bosons with integer spins. The elementary fermions have three families, each consisting of a pair of quarks and a pair of leptons and all of them have antiparticles of opposite charges, but otherwise identical properties. The leptons can propagate, but the quarks are bound in hadrons: the baryons (like the proton and neutron) consist of three quarks and the antibaryons of three antiquarks, and the mesons (like the pion) are bound states of a quark and an antiquark.

The three basic interactions in the Standard Model are derived from local gauge invariances: the strong interaction from a local $\mathrm{SU}(3)$ and the electroweak one from a local $\mathrm{U}(1) \otimes \mathrm{SU}(2)$ gauge invariance with the spontaneous symmetry breaking. These interac-

(C) D. HORVÁTH, 2019

ISSN 2071-0194. Ukr. J. Phys. 2019. Vol. 64, No. 7 tions are mediated by elementary bosons, the strong interaction by the eight gluons carrying the colour charges of the quarks and antiquarks, the weak interaction by the three heavy weak bosons, $W^{ \pm}$and $Z^{0}$ and the electromagnetism by the $\gamma$ photon. These bosons are virtual when they mediate the interactions, but they can also be emitted and observed experimentally, even the heavy weak bosons and the coloured gluons in high-energy collisions.

\section{CPT Invariance}

According to the well-known theorem of Emmy Noether, continuous symmetries of the Lagrangian lead to conservation laws. The conservation of the electric charge and of the fermion number is connected to the U(1) symmetry of the Dirac Lagrangian: that is a valid, non-breaking symmetry. The colourSU(3) symmetry of quantum chromodynamics leads to the conservation of the colour charge. The ultimate symmetry of matter and antimatter is manifested by the CPT invariance, which makes it possible to treat free antiparticles as particles moving backward in space and time. This is a most important symmetry of Nature: the physical laws do not change when charge $(C)$, space $(P)$ and time $(T)$ are simultaneously inverted: 


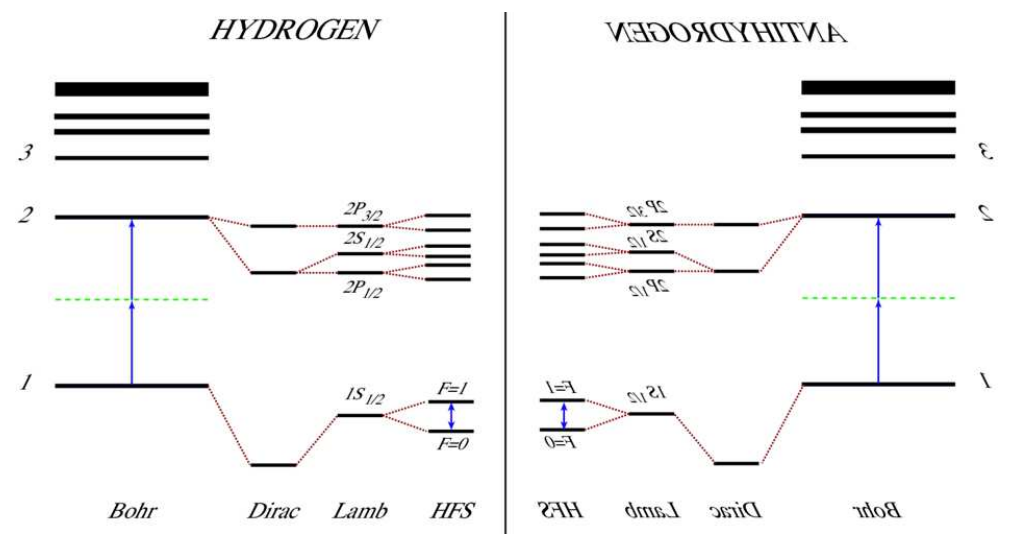

Fig. 1. Energy levels of hydrogen and antihydrogen. The $2 \mathrm{~S}-1 \mathrm{~S}$ transition offers extremely precise two-photon spectroscopy [3]

- charge conjugation (i.e. changing particles into antiparticles), $C \psi(r, t)=\bar{\psi}(r, t)$;

- parity change (i.e. mirror reflection), $P \psi(r, t)=$ $=\psi(-r, t)$, and

- time reversal, $T \psi(r, t)=\psi(r,-t) K$ where $K$ denotes complex conjugation.

Time reversal is an anti-unitary operation due to the phase factor connecting time and energy in the state function. As a result, $C P T$ is also anti-unitary, it conjugates the phase of the system while not changing the measurable properties. Using CPT invariance positron annihilation can be described as if an electron arrived, irradiated two or three photons and left backward in space and time.

$C P T$ invariance is supported by all known theoretical and experimental evidence. Its role is so fundamental in quantum field theory that it is almost impossible to test experimentally: in the case of observing a small deviation one should suspect the violation of a conservation law rather than $C P T$ violation. Giving up $C P T$ invariance brings dire consequences: one may lose causality, unitarity or Lorentz invariance. Nevertheless, it seems to be grossly violated: according to the generally accepted Big Bang theory of cosmology, at the end of the radiation period particles and antiparticles should have been produced in exactly the same amounts, but we cannot see antimatter galaxies anywhere [2]. This badly necessitates testing the $C P T$ invariance experimentally. To date the most precise one of such tests is the mass difference between the neutral kaon and anti-kaon as measured using kaon oscillation: the relative difference is less than $10^{-18}$.

\section{Antimatter Problems}

In 1928 Paul M. Dirac tried to produce a linear equation for the hydrogen atom and got two solutions for the electron: an ordinary one and another one with positive charge and negative mass. Dirac first assumed the latter non-physical, but three years later Carl Anderson observed positively charged electrons, positrons in cosmic rays (both of them were awarded the Nobel Prize).

In addition to the mysterious lack of antimatter in our Universe, there are some other questions for antiparticles. Is it really true that particles and their antiparticles have exactly the same properties except for the sign of their charges? Could there be a tiny difference between particle and antiparticle to cause the lack of antimatter galaxies? Are there particles which are their own antiparticles (called Majorana particles)? In principle, the neutrinos can be Majoranaparticles, although there are no signs of this in experiment. Could the dark matter of the Universe consist of such particles?

The above problems may point to a possible $C P T$ violation, and so we are obliged - in spite of our belief in its validity - to test $C P T$ invariance. The easiest way is to compare the properties of particles and antiparticles. In addition to the kaon-anti-kaon mass difference one can compare the spectroscopic properties of atoms and anti-atoms. It was shown [3] that the simplest and most precise such measurement with antiprotons should be to perform two-photon spectroscopy on antihydrogen atoms, $\overline{\mathrm{H}}=\left[\overline{\mathrm{p}} \mathrm{e}^{+}\right]$, the bound state of an antiproton and a positron, and that antihydrogen can be produced and confined in 
electromagnetic traps. The $1 \mathrm{~S}-2 \mathrm{~S}$ transition of antihydrogen (Fig. 1) seemed to be most eligible as it can be excited with two photons only and as a result of that it has a very long lifetime and consequently very narrow line width. Moreover, when applying two counter-propagating laser pulses one excludes the longitudinal Doppler-broadening of the line width, significantly increasing the precision of the measurement.

\section{The Antiproton Decelerator of CERN}

Antihydrogen atoms were first produced at the Low Energy Antiproton Ring (LEAR) at CERN and later also at Fermilab $[4,5]$. Relativistic antiprotons collide in the storage ring with $\mathrm{Xe}$ atoms and produce electron-positron pairs. With a low probability the antiproton can pick up a fast positron forming an antihydrogen atom which is neutral and leaves the ring along a straight beam line. The positron and the antiproton of the antihydrogen atom are then separated and identified: the positron annihilates to two photons and the antiproton to several charged pions.

CERN, the joint European Particle Physics Laboratory has built the Antiproton Decelerator, $A D$ facility (it is now called Antimatter Factory) in 199799 to study antimatter physics and to test the $C P T$ invariance, mainly via producing and studying antihydrogen. At the moment there are six experiments: three to test $C P T$ and another three to check antigravity, i.e. to measure the gravitational mass of the antiproton.

The Antiproton Decelerator works the following way. The Proton Synchrotron shoots protons with a $26 \mathrm{GeV} / c$ momentum onto an iridium target producing proton-antiproton pairs. From there the AD gets antiprotons of $3.57 \mathrm{GeV} / c$ momentum and slows them down to $100 \mathrm{MeV} / \mathrm{c}$ (corresponding to $5.3 \mathrm{MeV}$ kinetic energy) in four steps, in the first two steps with stochastic and then electron cooling [7]). The AD delivers $3 \ldots 4 \times 10^{7}$ antiprotons at $100 \mathrm{MeV} / c$ momentum to several experiments, which trap them in electromagnetic fields after suitable further deceleration, and using slow positrons make antihydrogen $\left(\overline{\mathrm{p}} \mathrm{e}^{+}\right)$ atoms [6].

The antiprotons have to slow further down to $\mathrm{keV}$ energies in order to facilitate trapping. The ALPHA and ATRAP experiments prepare spectroscopy on trapped antihydrogen, ASACUSA and BASE compare the properties (mass, charge and magnetic mo- ment) of protons and antiprotons at high precision, AEGIS and GBAR plan to measure the gravitational mass of antihydrogen, and ACE studied the effects of antiprotons on living tissue. The success of the AD experiments persuaded CERN to increase their efficiency by building a small storage ring ELENA (Extreme Low Energy Antiprotons) which will supply an order of magnitude higher number of slow antiprotons for trapping than the $\mathrm{AD}$ with the energy absorbers of the experiments. ELENA has been constructed and it will serve all AD experiments from 2020 on.

\section{Antihydrogen Production}

In order to produce antihydrogen, one has to confine both antiprotons and positrons in a trap, cool them to very low temperatures and then let them interact. The radiative recombination, $\left(\overline{\mathrm{p}} \mathrm{e}^{+} \gamma\right)$, should produce deeply bound atoms, but it is hopelessly slow. At the moment all AD experiments produce $\overline{\mathrm{H}}$ atoms using the three-body recombination reaction [8]: $\overline{\mathrm{p}} \mathrm{e}^{+} \mathrm{e}^{+} \rightarrow \overline{\mathrm{H}} \mathrm{e}^{+}$where a second positron carries away the released energy and momentum. This reaction has a quite high cross section, but it produces highly excited $\overline{\mathrm{H}}$ atoms which then should be de-excited to make spectroscopy possible.

Another method [9] is investigated at the $\mathrm{AD}: \overline{\mathrm{H}}$ production in collisions of antiprotons with positronium, the bound state of an electron and positron. This reaction has a high rate and results in nottoo-highly excited $\overline{\mathrm{H}}$, but it is more complicated to prepare.

The first cold, confined $\overline{\mathrm{H}}$ atoms were produced by the ATHENA experiment at the $\mathrm{AD}$, and its successor, the ALPHA (Antimatter Laser PHysics Apparatus) Collaboration made all steps leading from $\overline{\mathrm{H}}$ atoms confined in a trap, to their de-excitation and spectroscopy. At the same time the ASACUSA (Atomic Spectroscopy And Collisions Using Slow Antiprotons) Collaboration managed to produce and extract an $\overline{\mathrm{H}}$ beam from a trap.

\section{6. $\overline{\mathbf{H}}$ Spectroscopy by the ALPHA Experiment}

The ALPHA (Antimatter Laser PHysics Apparatus) Collaboration was the first and to date the only experiment to perform $2 S-1 S$ spectroscopy on antihydrogen [12]. The measurement was quite elaborate, developed gradually step by step in ten years: 
1. 90,000 antiprotons were captured and cooled in a Penning trap.

2. Mixed them with 3 million cold positrons and $50,000 \overline{\mathrm{H}}$ atoms were produced.

3. The remaining charged particles were removed by dropping the trapping potential.

4. $20 \overline{\mathrm{H}}$ atoms were stored in an inhomogeneous magnetic field at $T=0.54 \mathrm{~K}$ temperature.

5 . The $\overline{\mathrm{H}}$ atoms were kept trapped for $10 \mathrm{~s}$ in order to let them to undergo de-excitation to the $1 S$ ground state.

6. The excitation $1 S \rightarrow 2 S$ was performed with two $243 \mathrm{~nm}$ photons (standing wave for $300 \mathrm{~s}$ ) tuned around the resonance (appearance measurement).

7. A microwave irradiation removed the residual $1 S$ atoms (disappearance measurement).

8. The trap was flushed by dropping the confining $B$ field measuring the number of remaining $\overline{\mathrm{H}}$ atoms.

The $10 \mathrm{~s}$ waiting time was necessary to let the $\overline{\mathrm{H}}$ atoms de-excite to $1 \mathrm{~S}$, and at the same time short enough not to lose them from the trap as demonstrated by the ALPHA experiment earlier. Half the cold $\overline{\mathrm{H}}$ atoms can be confined depending on the spin polarization of the positron, and thus they can be flushed out by a microwave irradiation on resonance with the positron spin flip. This hyperfine transition was also studied when preparing the experiment [11]. At each step the antiproton annihilations were detected with checking the vertex positions of the events to make sure that they do not come from hitting the walls of the vessel. The last three steps made both an appearance and a disappearance measurement of the same reaction, and the results agreed with each other and also with the simulation assuming CPT invariance. At laser spectroscopy the laser power affects the result: that was also measured and the results normalized to the power of $1 \mathrm{~W}$. At last, ALPHA has managed also to observe the $1 \mathrm{~S}-2 \mathrm{P}$ Lyman alpha transition in antihydrogen [12].

Using $15000 \overline{\mathrm{H}}$ atoms the ALPHA experiment [10] yielded $2466061103079.4 \pm 5.4 \mathrm{kHz}$ for the $1 \mathrm{~S}-2 \mathrm{~S}$ transition frequency of antihydrogen. Its precision is just one order of magnitude behind that of ordinary hydrogen: $2466061103080.3 \pm 0.6 \mathrm{kHz}$. This means a confirmation of CPT on the level of $2 \times 10^{-12}$.

\section{Antimatter Gravity Measurement}

The negative mass of antiparticles in the Dirac theory keeps exciting the general public, although the masses of our everyday objects are mostly (about $95 \%$ ) energy-related. Of course, this is also something that has to be checked experimentally as precisely as possible. Unfortunately, gravity is so much weaker than electromagnetism that it makes a measurement with charged particles hopeless. There are nice gravity measurements made with neutrons, but the problem with antineutrons is that they cannot be slowed down without fast annihilation. That leaves antihydrogen for such studies. Of course, CPT invariance does not prescribe identical acceleration in Earth's gravitational field for protons and antiprotons: that is a result of the weak equivalence principle.

Testing antimatter gravity is the main aim of two AD experiments, AEGIS and GBAR. AEGIS (Antihydrogen Experiment: Gravity, Interferometry, Spectroscopy) is the largest AD collaboration (although still two orders of magnitude smaller than the largest LHC experiments). They are preparing to measure the gravitational falling of a beam of collimated $\overline{\mathrm{H}}$ atoms as compared to light using Moiré deflectometry. AEGIS will produce antihydrogen using the collisions of antiprotons with excited positronium atoms [13].

The GBAR (Gravitational Behaviour of Antihydrogen at Rest) Collaboration [14] plans to do an antihydrogen free-fall measurement. They plan to use such a dense positronium cloud that the antiprotons would pick up two positrons in two collisions to form an $\overline{\mathrm{H}}^{+}$ion which then can be cooled in several steps down to the vicinity of $10 \mu \mathrm{K}$ and they will move very slowly. Removing the excess positron via laser excitation they plan to let the neutral $\overline{\mathrm{H}}$ fall in the gravitational field of Earth and measure its acceleration. GBAR was the first AD experiment to use the slow antiprotons from ELENA in 2018. The ALPHA Collaboration has also constructed a free-fall apparatus for measuring antihydrogen gravity.

\section{Antiproton Properties}

The ASACUSA (Atomic Spectroscopy And Collisions Using Slow Antiprotons) Collaboration stopped antiprotons in helium gas and using laser spectroscopy measured the transition energies of antiprotons between atomic orbits determined the mass of the antiproton [15]. The method is based on the earlier observation that about $3 \%$ of antiprotons stopped in helium gas get captured in a metastable three-body bound state $\left[\overline{\mathrm{p}} \mathrm{He}^{+} \mathrm{e}^{-}\right]$. When a laser resonance ex- 
cites its transition to a non-metastable state, the antiproton will immediately annihilate. The experiment managed to increase the relative precision of the measurements from year to year, reaching the order of $10^{12}$ (meaning a CPT-test of similar precision) using two-photon spectroscopy [16] and buffergas cooling [17]. The ASACUSA Collaboration has built a post-decelerator system (essentially a radiofrequency quadrupole accelerator cavity working the opposite way) which increased the trapping efficiency by orders of magnitude and made it possible to produce an extracted beam [18] of slow antihydrogen atoms.

The BASE (Baryon Antibaryon Symmetry Experiment) performed direct high-precision measurements of the charge-to-mass ratio [19] and the magnetic moment [21] of a single antiproton stored in a cryogenic Penning trap. Both of them are, of course, sensitive $C P T$-tests when compared to those of the proton. It is remarkable, that this method is not destructive: in all 2016 BASE used 18 antiprotons for their measurements [20]. The obtained charge-to-mass ratio agrees with the predictions of the Standard Model (i.e. CPT invariance) at the level of $10^{-10}$. Moreover, assuming $C P T$ invariance the above result helps to confirm the weak equivalence principle [20] in Earth's gravitational field on the level of $6.8 \times 10^{-7}$.

\section{Antimatter in Space}

To solve the problem of the lack of antimatter galaxies, CERN prepared a cosmic detector, the Alpha Magnetic Spectrometer (AMS2) with the leadership of Nobel laureate Samuel Chao-chung Ting. It has a $1200 \mathrm{~kg}$ permanent magnet and it was launched in 2011 from the USA. It is placed onto the International Space Station and checks antiparticles in cosmic rays and also searches for dark matter annihilation. So far it did not detect anti-helium atoms, but saw many high-energy positrons which could come from pulsars or dark matter.

\section{Conclusion}

The Antiproton Decelerator of CERN was built 20 years ago in order to test the validity of $C P T$ invariance, the principle of matter-antimatter symmetry. The work of these two decades yielded many results, and the measured antiproton charge, mass, and magnetic moment, and the antihydrogen 1S-
$2 \mathrm{~S}$ transition measurement all conform the validity of $C P T$ and the Standard Model. The lack of antimatter galaxies seems to question $C P T$ invariance, but the results of the AMS2 space detector also confirmed: no anti-helium atoms are seen in cosmic rays. Thus $C P T$ invariance seems to be at absolute validity.

This work was supported by the National Research, Development, and Innovation Fund of Hungary under contracts K124850 and K128786.

1. F. Wilczek. In search of symmetry lost. Nature 433, 239 (2005).

2. A.G. Cohen, A. De Rujula, S.L. Glashow. A matter - antimatter universe? Astrophys. J. 495, 539 (1998).

3. M. Charlton, J. Eades, D. Horvath, R.J. Hughes, C. Zimmermann. Anti-hydrogen physics. Phys. Rept. 241, 65 (1994).

4. G. Baur et al. Production of anti-hydrogen. Phys. Lett. B 368, 251 (1996).

5. G. Blanford et al. [E862 Collaboration]. Observation of atomic anti-hydrogen. Phys. Rev. Lett. 80, 3037 (1998).

6. J.S. Hangst. Fundamental physics with antihydrogen. Springer Tracts Mod. Phys. 256, 203 (2014).

7. P. Beloshitsky. Report on operation of antiproton decelerator. AIP Conf. Proc. 821 (1), 48 (2006).

8. G. Gabrielse, S.L. Rolston, L. Haarsma, W. Kells. Antihydrogen production using trapped plasmas. Phys. Lett. A 129, 38 (1988).

9. B.I. Deutch, F.M. Jacobsen, L.H. Andersen, P. Hvelplund, H. Knudsen, M.H. Holzscheiter, M. Charlton, G. Laricchia. Anti-hydrogen production by positronium anti-proton collisions in an ion trap. Phys. Scripta T 22, 248 (1988).

10. M. Ahmadi et al. Characterization of the $1 \mathrm{~S}-2 \mathrm{~S}$ transition in antihydrogen. Nature 557, 71 (2018).

11. M. Ahmadi et al. [ALPHA Collaboration]. Observation of the hyperfine spectrum of antihydrogen. Nature 548, 66 (2017).

12. M. Ahmadi et al. [ALPHA Collaboration]. Observation of the $1 \mathrm{~S}-2 \mathrm{P}$ Lyman- $\alpha$ transition in antihydrogen. Nature 561, 211 (2018).

13. C. Amsler et al. [AEgIS Collaboration]. Velocity-selected production of $2^{3} \mathrm{~S}$ metastable positronium. Phys. Rev. A 99, 033405 (2019).

14. B. Mansouli [GBAR Collaboration]. Status of the GBAR experiment at CERN. Hyperfine Interact. 240, 11 (2019).

15. R.S. Hayano, M. Hori, D. Horvath, E. Widmann. Antiprotonic helium and CPT invariance. Rept. Prog. Phys. 70, 1995 (2007).

16. M. Hori et al. [ASACUSA Collaboration]. Two-photon laser spectroscopy of antiprotonic helium and the 
antiproton-to-electron mass ratio. Nature $\mathbf{4 7 5}, 484$ (2011).

17. M. Hori et al. [ASACUSA Collaboration]. Buffer-gas cooling of antiprotonic helium to 1.5 to $1.7 \mathrm{~K}$, and antiprotonto-electron mass ratio. Science 354, 610 (2016).

18. Y. Nagata et al. Progress of antihydrogen beam production using a double cusp trap. JPS Conf. Proc. 18, 011007 (2017).

19. S. Ulmer et al. [BASE Collaboration]. High-precision comparison of the antiproton-to-proton charge-to-mass ratio. Nature 524, 196 (2015).

20. S. Ulmer, A. Mooser, H. Nagahama, S. Sellner, C. Smorra. Challenging the standard model by high-precision comparisons of the fundamental properties of protons and antiprotons. Phil. Trans. Roy. Soc. Lond. A 376, 20170275 (2018).

21. C. Smorra et al. [BASE Collaboration]. A parts-per-billion measurement of the antiproton magnetic moment. Nature 550, 371 (2017).

Received 08.07.19
Д. Хорват

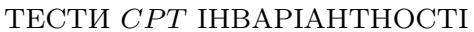

НА АНТИПРОТОННОМУ УПОВІЛЬНЮВАЧІ ЦЕРНу

$\mathrm{P}$ е $з$ ю м е

Стандартна Модель теорії елементарних частинок базується на симетріях: як структура композитних частинок, так i їх взаємодія виводяться з принципів калібровної інваріантності. Деякі з них, як парність та $C P$ симетрія, порушуються слабкою взаємодією, та навіть маси породжуються спонтанним порушенням симетрії. Згідно з CPT інваріантності - найсуттєвішої симетрії Стандартної Моделі - матерія та антиматерія еквівалентні. Проте, через відсутність антиматерії у Всесвіті, цю симетрію постійно вивчають у ЦЕРНі. Ми даємо огляд цих експериментів: вимірюємо властивості антипротонів у порівнянні з протонами на Антипротонному Уповільнювачі, а також шукаємо антиречовину в космічних променях. 